McElroy, T., Seta, J. J., \& Waring, D. (2007). Reflections of the Self: How Self-esteem Imposes onto Risky-choice Framing Tasks. Journal of Behavioral Decision Making, 20: 223-240. Published by Wiley InterScience (ISSN: 0894-3257). DOI: 10.1002/bdm.551 The definitive version is available at www3.interscience.wiley.com

\title{
Reflections of the Self: How Self-Esteem Determines Decision Framing and Increases Risk Taking
}

Todd McElroy, John J. Seta, and Douglas A. Waring

\begin{abstract}
Historically, research examining the influence of individual personality factors on decision processing has been sparse. In this paper we investigate how one important individual aspect, self-esteem, influences imposition and subsequent processing of ambiguously, negatively or positively framed decision tasks. We hypothesized that low self-esteem individuals would impose a negative frame onto ambiguous decision problems and would be especially sensitive to negatively framed decision tasks. In Study 1 we utilized a self-framing procedure and demonstrated that HSE participants were evenly divided in the hedonic valence they self-imposed whereas LSE participants were more likely to self-impose a negative frame. When these differences were accounted for, HSE and LSE participants were equivalent in risk seeking/avoiding choices. Study 2 used a risky-choice framing task and found that LSE individuals were especially sensitive to the negative frame. Study 3, provided converging evidence and generalization of these findings to a reflection tasks involving money.
\end{abstract}




\section{INTRODUCTION}

When faced with numerically equivalent options, does the mode of presentation affect an individual's choice? Over two decades of research suggests that it does and this phenomenon is now commonly referred to as the framing effect. The framing effect has emerged as the foremost example of "irrational" decision-making. Yet, during the period there has been increasing calls for a more complete explanation that emphasizes the processing of decision tasks and less focus on the apparent shortcomings and violations of human decision-making. In order to address these concerns, some researchers have sought to capture a more stable picture of choice preference by focusing on internalized aspects of the decision maker that come to bear on the decision process (e.g., Lauriola, Russo, Lucidi, Violani, \& Irwin, 2005; Levin, Gaeth, Schneider, \& Lauriola, 2002; Reyna \& Brainerd, 1991; Stanovich \& West, 1998). Identifying these stable individual differences should lead to a better understanding and predictability of human decision-making.

We begin by discussing the framing effect, its theoretical foundations and related research.We then review studies showing that individual personality factors can influence decision-making, focusing specifically on the framing effect. Next, we discuss related research involving the personality construct of self-esteem. Finally, we argue that if this positive or negative "self valence" created by self-esteem influences the perceived valence of a decision task, then this should have an influence on subsequent decision processing. This creates a situation where internally positive or negative aspects of the decision-maker influence the perceived external positive or negative attributes (i.e., the frame) of the decision problem.

\section{The framing effect}

Since Kahneman and Tversky's (1979) introduction of prospect theory, a great deal of research has attempted to better understand the generalizability and potential moderators of the framing effect. Methods for testing the framing effect have varied. However, the most widely used and tested example is the Asian disease problem (Tversky \& Kahneman, 1981). In the Asian disease problem, all participants are first given the same introductory scene and then provided with two alternatives from which to choose (See Table 1).

Because of the numerical equivalence in magnitude of the two options, there should be no difference in preference for people who are risk neutral. Also, there should be no shifting of preferences from risk seeking to risk avoiding simply because of how the problem is framed. In fact, numerous demonstrations have shown that people do shift their preferences based upon how the alternatives are framed. According to prospect theory, people tend to prefer the risk-averse option when the problem is framed positively, or in terms of gains and the risk-seeking option when the problem is framed negatively, or in terms of losses.

According to prospect theory, this shifting of preference occurs because outcomes are represented differently depending upon whether they are expressed as positive "gains"' or negative "losses." The value of the prospect is represented by an s-shaped value function that is concave for gains and convex for losses. The concave-convex nature of the value function allows for the prediction that, when confronted with options of equal expected value, the alternative that is risk-free should have more subjective value when the problem is framed positively whereas the risky option should have relatively more subjective value when the problem is framed negatively. Thus, prospect theory makes the prediction that, when faced with a decision framed in terms of potential gains, individuals should tend to be risk-averse and when faced with a decision framed in terms of potential losses, they should tend to be risk seeking.

\section{Framing research}

Reviews of the framing literature by Kuhberger (1998) and Levin, Schneider, and Gaeth (1998) brought together a large number of studies across different disciplines. Kuhberger, through meta-analysis, demonstrated that the framing effect for risky-choice type problems is small to moderate in strength $(d=0.31)$. This seems even more substantial in light of the large variation of procedural differences that exist in framing research. Levin et al., (1998) provide theoretical clarity for some of the disparate ways that researchers have constructed the framing task. Specifically, Levin et al., identify three types of framing tasks that have emerged: goal, attribute and risky-choice framing. The most widely tested type, risky-choice, is also the type of framing task that produces the strongest and most consistent framing effects. The typical risky choice framing task involves three defining features. First, participants are presented with a problem that has two options, one is a certain option (risk-averse) and the other involves a probability of occurrence (risk seeking). Secondly, the problem is framed positively, usually in terms of gains or negatively, usually in terms 
of losses. Third, within each frame, each of the options are of the same expected value, or numerically equivalent.

Table 1. The Asian disease problem framed as gains and losses

\begin{tabular}{|c|c|c|}
\hline Problem & Gains & Losses \\
\hline $\begin{array}{l}\text { Imagine that the U.S. is preparing for the } \\
\text { outbreak of an unusual Asian disease, which } \\
\text { is expected to kill } 600 \text { people. Two altemative } \\
\text { programs to combat the disease have been } \\
\text { proposed. Assume that the exact scientific } \\
\text { estimates of the consequences of the programs } \\
\text { are as follows: }\end{array}$ & $\begin{array}{l}\text { If program A is adopted, } \\
200 \text { people will be saved. } \\
\text { If Program B is adopted, } \\
\text { there is a one-third probability } \\
\text { that } 600 \text { people will be saved } \\
\text { and a two-thirds probability } \\
\text { that no people will be saved. }\end{array}$ & $\begin{array}{l}\text { If program A is adopted, } \\
400 \text { people will die. } \\
\text { If Program B is adopted, } \\
\text { there is a one-third probability } \\
\text { that nobody will die and a } \\
\text { two-thirds probability that } \\
600 \text { people will die. }\end{array}$ \\
\hline
\end{tabular}

Research investigating the framing effect has yielded significant insight while creating issues of controversy. For example, researchers have investigated how elaborating on a decision problem by methods such as, spending more time or effort, can have an effect on framing. Some research has shown support for the idea that inducing elaboration will attenuate the framing effect (McElroy \& Seta, 2003; Miller \& Fagley, 1991; Sieck \& Yates, 1997; Simon, Fagley, \& Halleran, 2004; Takemura, 1994) whereas other findings suggest that it may actually enhance or have no effect at all (Bloomfield (in press); Fagley \& Miller, 1987; Igou \& Bless, in press; LeBoeuf \& Shafir, 2003). Other research has found that presenting the framing task in the context of a statistical or medical decision problem (Bless, Betsch, \& Franzen, 1998) or different scenario situations (Fagley \& Miller, 1997; Schneider, 1992) influences choice preference. Researchers taking a dual-systems approach also have demonstrated that different systems of processing tend to yield different results for framing tasks (e.g., Igou \& Bless, in press; McElroy \& Seta, 2004; Reyna \& Brainerd, 1991; Wang, 2005).

\section{Personality influences on framing}

One avenue of investigation that may help provide a better understanding of framing effects is to study how individual differences in personality traits influence the processing of information in a framed decision task. Tversky and Kahneman (1981, p. 453) vividly point this out suggesting that: "The frame that a decision maker adopts is controlled partially by the formulation of the problem and partly by the norms, habits and personal characteristics of the decision-maker.' 'This theme has been echoed by later researchers investigating issues central to the framing effect (e.g., Kuhberger, 1998; Levin, Gaeth, Schneider,\& Lauriola, 2002; Stanovich \& West, 1998, Stanovich \& West, 2000; Tversky \& Kahneman 1981). Therefore, there seems to be a general consensus among decision-making researchers that it would be short sighted to believe that a decision-makers' representation of the problem is only derived from the external information provided within the task. Rather, most researchers agree that certain internal aspects of the person can and do influence the frame that decision-makers adopt (e.g., Bartels, 2006; Kuhberger, 1998; Lauriola et al. 2005; Levin et al. 2002; McElroy \& Seta, 2006; Reyna, Lloyd, \& Brainerd, 2003; Stanovich \& West, 1998, 2000; Tversky \& Kahneman, 1981). Yet, while most researchers agree that this line of research is crucial for a complete understanding of decision processing, studies specifically designed to investigate how individual differences influence choices in framed decision tasks have been limited, with some notable exceptions.

One personality construct that has generated a significant amount of research interest, as well as controversy, is that of need-for-cognition (NFC). Some research has suggested that the decisions of high NFC individuals are less likely to be influenced by the frame relative to low NFC individuals. This is said to occur because high NFC individuals process the information more thoroughly and perceive the numerical equalities of the options (e.g., Chatterjee, Heath, Milberg,\&France, 2000; Smith\&Levin, 1996; Zhang\&Buda, 1999). Other research has suggested that NFC plays no role in determining whether or not the framing effect is likely to occur (LeBoeuf \& Shafir, 2003; Levin et al. 2002). Recently, Simon, Fagley, and Halleran (2004) have demonstrated that both high NFC and depth of processing are necessary for more thorough processing of the alternatives and consequently, a moderation of the framing effect. 
Other personality constructs also have proven beneficial for understanding the framing effect. For example, Fagley and Miller (1990) found no influence for the personality construct of field dependence/ independence. However, they noted that the personality trait of risk seeking seemed to play a role in how gender differences influenced framing. Lauriola and Levin (2001) explored potential influences of the Big-5 personality traits. They found that some of the Big-5 personality traits have different effects on risky-choice depending upon whether the options are presented within the positive or negative frames and that individuals classified as high in neuroticism exhibited an enhanced framing effect. Levin, Gaeth and Schreiber (2002) found that a subset of both the Big-5 and the Faith in Intuition inventory were related to individual risk seeking preferences for a typical framing task.

Another avenue for investigating individual differences in risky-choice decision tasks is to study how individuals vary in the hedonic tone that they spontaneously self-impose onto risky-choice decision tasks. Empirical evidence suggests that adult individuals are relatively consistent in the subjective frame that they impose upon related decision tasks (e.g., Elliott \& Archibald, 1989). Recently, Wang (2004) investigated the issue of frame imposition in a typical risky-choice paradigm by developing an ambiguous version of the risky-choice decision task which allowed participants to self-impose (create) the positivity or negativity of the framing task. This novel design allowed Wang to ascertain that, when faced with a task of ambiguous hedonic tone, individuals will vary in the positivity/negativity they impose. Further, Wang also found that participants tended to show a bias toward positive framing, a finding consistent with the literature on belief (Gilbert, 1991), linguistic bias (e.g., Clark \& Chase, 1972) and predictions derived from fuzzy-trace theory (Reyna \& Brainerd, 1991).

\section{Self-esteem}

One characteristic of human nature is the unfolding of a particular evaluation of ones' "self'" as a person. This evaluative sense that we humans possess is referred to as self-esteem. Self-esteem is seen as a relatively enduring characteristic (Trzesniewski, Donnellan, \& Robins, 2003) that carries with it both motivational and cognitive components (Baumeister, 1993). Some theorists have described self-esteem as a judgmental process, comparing how one stands relative to other people (e.g., Coopersmith, 1967; Rosenberg, 1979) and more recently as a barometer of group acceptance (Leary \& Baumeister, 2000). Other theorists have defined self-esteem more as an outcome of a developmental processes that is largely focused on the primary caregiver (e.g., Sullivan, 1953). While there is some controversy about the development and function of self-esteem, there is general agreement that the construct of self-esteem can be either positive or negative and is derived from a favorable or unfavorable attitude toward the self or "self regard" (Rosenberg, 1965).

\section{Overview of studies 1-3}

Research investigating self-esteem has offered many insights into human behavior especially addressing questions of how high self-esteem (hereafter HSE) and low self-esteem (hereafter LSE) individuals may respond to evaluation and competition. Because the research on self-esteem is so diverse, we shall focus on studies that are particularly relevant to our question of how perceivers' level of self-esteem influences their decisions. We concentrated on studies whose procedures and subject matter seem to be generalizable to the decision tasks used in the present paper.

There is evidence that, under certain circumstances, LSE individuals may be more negatively focused than their HSE counterparts. For example, LSE individuals are especially likely to interpret an ambiguous remark as an insult (Schlenker, 1980). In addition, Bellavia and Murray (2003) found that LSE participants responded more to their partners when they were in either a neutral or negative mood state whereas HSE individuals were relatively unaffected by their partner's mood state (see also Koch, 2002).

If LSE individuals do have a tendency to interpret ambiguous information more negatively then they also may self-impose a negative frame onto an ambiguously framed decision problem. Study 1 was designed to test the validity of this hypothesis. In addition, it also may be the case that because of their negative focus, LSE individuals are especially susceptible to negatively framed tasks and the risk-seeking decision they tend to promote (Kahneman \& Tversky, 1979). If so, then they should be more risk-seeking than HSE individuals when the decision task is framed negatively (or as a loss). 
It is less clear if and how LSE and HSE individuals will differ on positively framed tasks. However, from the research by Bellavia and Murray (2003), it may be the case that LSE and HSE individuals will not differ significantly in their susceptibility to positively framed tasks. As previously noted, the researchers found that LSE participants responded more to their partner's negative and neutral moods whereas HSE participants were relatively unaffected by mood. If these results can be generalized to framing tasks then we can conclude that, because LSE individuals were not especially influenced by the positive mood of their partner and because HSE individuals were relatively unaffected by any of their partner's mood states, then neither LSE nor HSE individuals will be especially susceptible to positively framed tasks.

\section{Summary of predictions}

Using findings from prior studies as a backdrop for our predictions, we expect LSE individuals to self-impose an especially negative frame onto a decision problem that is framed ambiguously. This prediction was the focus of Study 1.We also expect that when LSE and HSE individuals are confronted with decision tasks that are framed either positively or negatively, they should differ in their sensitivity to the negative (loss) frame but not the positive (gain) one. The net effect of this difference is more risk-seeking choices for LSE individuals when the decision problem is framed negatively and no difference in the risk-seeking choices of LSE and HSE individuals when it is framed positively.

In Study 2, we used a traditional risky-choice framing task to determine whether LSE and HSE individuals differ in their sensitivity to positively and negatively framed tasks. Study 3 tested whether findings similar to those obtained in Study 2 would be obtained with a reflection problem, rather than a risky-choice framing one.

\section{STUDY 1}

\section{Method}

\section{Participants and design}

One-hundred and sixty-two individuals including 68 males, 92 females and 2 non-gender reporting participants, took part in our study. In return for their participation in our experiment, participants received credit toward their introductory psychology class research requirement. The study examined level of self-esteem (high, low) and self-imposed framing (positive, negative).

\section{Materials and procedure}

We first assessed individual levels of self-esteem by asking participants for their ratings on the Rosenberg self-esteem scale (Rosenberg, 1965). The Rosenberg self-esteem scale consists of 10 self-esteem related items such as "on the whole I am satisfied with myself", and "all in all, I am inclined to feel that I am a failure.” The scale for each item was presented in a 4-point Likert format. The self-esteem score was determined by adding the five positive items to the reverse score of the five negative items. After participants had completed the Rosenberg self-esteem scale, they were instructed to turn the paper face down in front of them.

Next, participants were presented with our self-framing task. The content of this task is very similar to other self-framing tasks developed by Wang (2004 Study 2). Our task differed slightly. We modified the wording to correspond to that used in the traditional Asian disease problem.

The introduction to the self-framing problem was exactly the same as that used in the traditional Asian disease problem (Tversky \& Kahneman, 1981). Following the introduction however, the alternatives were presented in a much different manner. Consistent with Wang (2004 Study 2), we first presented participants with a whole-pie chart (see Appendix 1). Directly after presentation of the pie charts, the following statement appeared: "Based on your interpretation of the pie display of the expected outcomes, complete the following sentences in your own words." 
They were then presented with the following options:

If Plan A is adopted, people will

If Plan B is adopted, there is a one-third chance that people will and a two-thirds chance that people will

Following the presentation of these open-ended alternatives, participants were asked which option they would prefer. After participants had made their choice, they were again asked to turn the paper face down and indicate various types of demographic information. They were then debriefed about the procedures used in the study.

\section{Results and discussion}

To examine the results of our study we first wanted to obtain a reliable index of the hedonic frame that each participant self-imposed. Similar to Wang (2004), we created a composite of the hedonic frames that participants had generated for the Asian disease problem. We then obtained a sample of 14 undergraduates, 7 males and 7 females, from the same population. To determine their hedonic ratings of the self-generated frames, we informed them that they were evaluating words that their fellow students had used to describe the Asian disease problem. Then we asked them to evaluate these words on a 5-point scale ranging from unpleasant to pleasant. The hedonic level of each frame was determined by averaging across all 14 participants in our norming sample. Framing words that scored above neutral (3 on our scale) were considered as positive frames whereas those that scored below neutral were considered to be negative. The mean rating and frequency for each of the self-generated frames are listed in Appendix 2. For those participants who self-imposed either a purely negative or purely positive frame we found that $67 \%$ self-imposed a negative frame whereas $33 \%$ imposed a positive frame.1 A complete list of frames that participants generated can be found in Appendix 2. ${ }^{2}$

Table 2. Study 1: Number of positive and negative self-imposed frames as a function of self-esteem

\begin{tabular}{lcc}
\hline & \multicolumn{2}{c}{ Self-imposed frame } \\
\cline { 2 - 3 } & $\frac{\text { Positive frame }}{N(\% \text { total })}$ & $\frac{\text { Negative frame }}{N(\% \text { total })}$ \\
\hline Self-esteem: & $13(19)$ & $55(81)$ \\
$\quad$ Low & $30(48)$ & $32(52)$ \\
High & & \\
\hline
\end{tabular}

\section{Primary hypothesis investigation}

We first wanted to examine whether participants' level of self-esteem influenced their self-imposed framing of the Asian disease task. To answer this question, we performed a nominal logistic analysis with level of self-esteem as the independent variable and participants' self-imposed frame (positive or negative) as the dependent variable. In this analysis we only included those participants (130) who imposed a completely positive or negative frame.3 This analysis revealed that, overall, HSE and LSE individuals differed significantly in the frame they imposed on the Asian disease problem $x_{2}(1, N=130)=11.9, p<0.001$. We then performed a median split on participants' self-esteem scores which yielded a classification of 32 and below for LSE and 33 and above for HSE. As may be seen in Table 2, 81\% of LSE individuals self-imposed a negative frame onto the ambiguous alternatives. An additional nominal logistic test revealed that there was a significant difference between the percentage of LSE participants who imposed a negative (81\%) versus a positive frame $(1, \mathrm{~N}=68)=27.91, \mathrm{p}<0.0001$. HSE individuals on the other hand, tended to be relatively equal in the valence of the frame that they imposed with only 52\% of these individuals imposing a negative frame. An additional nominal logistic analysis revealed that there was no difference in the number of HSE individuals who imposed a negative (52\%) versus positive (48\%) frame $\mathrm{x}_{2}(1, \mathrm{~N}=62)=0.06, \mathrm{p}>0.7$. Thus, HSE individuals as a group were even handed, with an equal number imposing a positive versus a negative frame. We also subjected the valences of participants' self-imposed frames to an ANOVA to determine 
whether there was a difference in the frames used by LSE and HSE individuals. To perform this analysis we obtained an average across the three imposition alternatives. It is important to note that we included frames from all participants - frames that were purely negative, purely positive and mixed. The ANOVA revealed a significant self-esteem effect, $F(1,160)=17.7, p<0.001$. LSE individuals' self-imposed frames were more negative $(M=1.9)$ than those generated by HSE individuals $(M=2.9)$.

\section{Choices within a self-imposed task}

We have shown that HSE and LSE individuals differ in the valence of frames they impose onto a typical framing task. However, we also wanted to determine whether HSE and LSE individuals differ in their decisions after they have self-imposed a frame onto the task. Specifically, did HSE and LSE participants differ in their risky-choice responses once they had imposed either a positive or negative frame onto the task? One way to test this question is by performing a nominal logistic analysis with level of self-esteem and frame as independent variables and choice as the dependent variable. This will also tell us whether a significant framing effect occurred in this study. That is, were participants who self-imposed the positive frame more likely to choose the risk-averse option whereas those who self-imposed the negative frame more likely to choose the risk-seeking option. The results indicated that there was no interaction between level of selfesteem and frame $x_{2}(1, N=130)=0.12, p>0.7$. This null finding demonstrates that HSE and LSE

participants did not have significantly different responses across the different frames. The analysis also revealed a significant framing effect $\mathrm{X}_{2}(1, \mathrm{~N}=130)=5.75, \mathrm{p}<0.05$. As may be seen from Table 3 , participants were generally risk-averse when they self-framed the task in a positive way and they were risk seeking when they framed it negatively.

Table 3. Study 1: Number of risk-a voidant and risk-seeking choices as a function of self-esteem, and self-imposed frame of the Asian disease problem

\begin{tabular}{|c|c|c|}
\hline & \multicolumn{2}{|c|}{ Choices } \\
\hline & Risk-a voidant & Risk-seeking \\
\hline & $N(\%$ total $)$ & $N$ (\% total) \\
\hline \multicolumn{3}{|c|}{$\begin{array}{l}\text { High self-esteem } \\
\text { Framing: }\end{array}$} \\
\hline Gains & $21(70)$ & $9(30)$ \\
\hline Losses & $14(44)$ & $18(56)$ \\
\hline \multicolumn{3}{|c|}{ Low self-esteem } \\
\hline \multicolumn{3}{|c|}{ Framing: } \\
\hline Gains & $8(62)$ & $5(38)$ \\
\hline Losses & $23(42)$ & $32(58)$ \\
\hline
\end{tabular}

To further investigate this point, we examined whether HSE and LSE participants had different responses within each of the positive and negative frames that they self-imposed. The results of this analysis revealed that there was no difference within the self-imposed positive $x_{2}(1, N=43)=0.29, p>0.59$ or self-imposed negative condition $\mathrm{x}_{2}(1, \mathrm{~N}=87)=0.03, \mathrm{p}>0.8$. This null finding provides direct evidence that HSE and LSE participants did not differ in their responses once they had self-imposed a positive or negative hedonic frame onto the task. In fact, as can be seen in Table 3, participants' choices, in light of the self-imposed frame, are very similar for both HSE and LSE participants.

Prior research has found that there may be gender differences in the strength of the framing effect (e.g., Fagley \& Miller, 1990; Wang, Simons, \& Bredart, 2001), making it a potentially important variable to consider in framing research (Fagley \& Miller, 1997). Further, there is also evidence that there may be a relationship between self-esteem and gender, with males reporting slightly higher self-esteem scores than females (e.g., Kling, Hyde, Showers, \& Buswell, 1999). Therefore, we wanted to examine the influence of gender in the results of our study. Consistent with prior research, we found that males $(M=33.5)$ had slightly higher self-esteem scores than females $(M=31.5)$. Within the levels of self-esteem, we can see that both 
genders were relatively well represented, with HSE consisting of (31=males, 31=females) and LSE having (24=males, 42=females). In order to examine potential gender effects, we performed a nominal logistic analysis ${ }^{4}$ with frame and gender as our independent variables and participants' choice as our dependent variable. The results revealed a significant main effect for problem framing $X^{2}(1, N=128)=4.81$, $\mathrm{p}<0.05$ and a non-significant main effect for gender $\mathrm{X}_{2}(1, \mathrm{~N}=128)=1.7, \mathrm{p}>0.2$ and the overall interaction $\mathrm{X}^{2}(1, \mathrm{~N}=128)=0.7, \mathrm{p}>0.39$.

\section{STUDY 2}

In Study 1 we examined how HSE and LSE individuals differed in the positive/negative valence they selfimposed onto a risky-choice type decision task and their subsequent choices. In Study 2 we examine how HSE and LSE individuals differ when presented with a risky-choice decision task that is framed positively or negatively. Specifically, LSE individuals should be especially sensitive to negatively framed decision tasks whereas HSE individuals should not differ in their sensitivity.

\section{Method}

\section{Participants and design}

One hundred-ten individuals participated in our experiment. Our sample consisted of 67 females, 40 males and 3 individuals who did not indicate gender. Each participant received class credit for their participation. The experimental design consisted of two levels of self-esteem (high, low) and the manipulated variable of frame (positive, negative) for the Asian disease task.

\section{Materials and procedure}

Similar to Study 1, we first assessed individual levels of self-esteem by asking participants for their ratings on the Rosenberg self-esteem scale (Rosenberg, 1965). After participants had completed the Rosenberg self-esteem scale, they were presented with the risky-choice framing task. The framing task used in this study was the original Asian disease problem (Tversky \& Kahneman, 1981). On the following page we asked participants to identify what frame was just presented to them (i.e., lives saved or lives lost). Next, participants were provided with the PANAS scale (Watson, Clark, \& Tellegen, 1988). The PANAS is a measure of current affect that consists of 20 adjectives. Participants rate, on a 5-point scale, the extent that each of the adjectives reflect how they feel. They were then instructed to turn the paper face down in front of them. After all participants had made their choices, they were asked to indicate their gender. They were then debriefed about the procedures used in the experiment.

\section{Results and discussion}

We performed a nominal logistic regression on participants' decisions to test whether both positive and negative framing conditions produced the predicted framing effect. This analysis yielded the common effect for framing $x^{2}(1, N=110)=13.94, p<0.001$.

As was the case in Study 1, we wanted to examine whether gender might be playing a role in the analysis of self-esteem. Similar to Study 1, we found that males $(M=33)$ had slightly higher self-esteem scores than females $(M=30.7)$ and that both genders were relatively well represented with both the HSE (23=males, 24=females) and LSE (17=males, 43=females). We again performed an analysis with gender and frame as our independent variables and participants' choice as our dependent variable. A significant main effect for framing emerged $x^{2}(1, N=107)=12.3$, $p<0.001$, no other effects were significant.

In order to investigate our hypothesis concerning self-esteem influences on framing tasks, we performed a nominal logistic regression using self-esteem and frame as our independent variables and participants' choices as our dependent variable.5 The results of this analysis revealed a significant effect for the framing conditions $X^{2}(1, N=110)=14.63$, $p<0.001$, as well as a significant self-esteem $x$ frame interaction $X^{2}(1, N=110)=4.01, p<0.05$. The Self-Esteem $x$ Frame interaction suggests that individuals with different self-esteem levels make different types of choices across framing tasks. As may be gathered 
from Table 4, the pattern of results appears to suggest that this effect is mainly due to the negative framing condition. In this condition, LSE participants were relatively more risk seeking. To determine whether this pattern of results is statistically meaningful, we performed an analysis within each of the framing conditions.

Table 4. Study 2: Number of risk-avoidant and risk-seeking choices as a function of self-esteem, and framing

\begin{tabular}{|c|c|c|}
\hline & \multicolumn{2}{|c|}{ Choices } \\
\hline & Risk-a voidant & Risk-seeking \\
\hline & $N(\%$ total $)$ & $N(\%$ total $)$ \\
\hline \multicolumn{3}{|c|}{ High self-esteem } \\
\hline \multicolumn{3}{|c|}{ Framing: } \\
\hline Gains & $15(60)$ & $10(40)$ \\
\hline Losses & $10(43)$ & $13(57)$ \\
\hline \multicolumn{3}{|c|}{ Low self-esteem } \\
\hline \multicolumn{3}{|c|}{ Framing: } \\
\hline Gains & $20(67)$ & $10(33)$ \\
\hline Losses & $5(16)$ & $27(84)$ \\
\hline
\end{tabular}

First, we performed a nominal logistic regression on LSE and HSE participants responses within the losses condition. The results of this analysis demonstrated that LSE and HSE individuals differed significantly in their choices $\mathrm{X}^{2}(1, \mathrm{~N}=55)=4.9, \mathrm{p}<0.05$. This suggests that, in the negative domain, LSE individuals are more likely to choose the risky option compared to HSE individuals. Next, we performed the same type of analysis for the positive condition. The results revealed no significant difference between HSE and LSE individuals $\mathrm{X}^{2}(1, \mathrm{~N}=55)=0.26, \mathrm{p}>0.6$.

To examine whether affect may be the causal factor rather than self-esteem we examined participants PANAS scores. Not surprisingly, positive affect was positively correlated with self-esteem $r(102)=0.42$, $p<0.001$ and negative affect was negatively correlated $r(102)=0.32, p<0.001$. In order to investigate how affect might be influencing participants' responses, we first tested whether there was an interaction with framing.6 This analysis revealed no significant interaction $\mathrm{F}(1,102)^{1 / 4} / 4.03$, $\mathrm{p}>0.8$ or main effect $\mathrm{F}(1,102)=1.4, \mathrm{p}>0.2$ for positive affect. We found similar results for negative affect; no significant interaction $F(1,102)=2.16, p>0.1$ and no main effect $F(1,102)=0.01, p>0.9$. We then tested the influence of affect within each of the framing conditions. We found that in the negative framing condition neither positive $F(1,49)=0.44, p>0.5$ nor negative affect $F(1,49)=1.03, p>0.3$ predicted participants' choices. In the positive framing condition we also found no significant effects for either positive $F(1,53)=1.12, p=0.3$ or negative affect, $F(1,53)=1.14, p>0$.2. The findings from this study suggest that when LSE and HSE individuals are confronted with a typical framing task, they do differ in their risky choices. However, this difference seems to be limited to the negative framing condition. In this condition LSE individuals are more likely to choose the risk-seeking option relative to HSE individuals. An analysis of the affect measure revealed that, within this type of framing situation, affect did not play a significant role in participants' risky-choice responses.

Subsequent to all other measures, participants were asked to recall the name of the frame (i.e., people saved or people die) that was contained in the decision task. Although almost everyone recalled the frame correctly, among those LSE participants who incorrectly recalled the frame, six out of seven incorrectly indicated a negative frame (people die) when the frame was indeed positive. HSE participants, however, were evenly divided, with two incorrect for each condition. Although this evidence is only anecdotal, it is congruent with the hypothesis that LSE individuals are more susceptible than their HSE counterparts to a negative frame. 


\section{STUDY 3}

In Study 2, we used a risky-choice decision task and found that LSE participants were more sensitive than their HSE counterparts to negatively valenced frames and, in turn, exhibited more risk-seeking preference in the negative framing condition. In Study 3 we further explore our hypothesis by examining HSE and LSE participants' gain/loss sensitivity under conditions of reflection.7

Framing effects refer to reversal of preferences based upon how alternatives are presented. In framing vignettes, such as the Asian disease problem, the domain remains consistent but the outcome is presented as to involve either positive or negative aspects of the outcome. Reflection effects differ from framing effects in two important ways. First, the alternatives across both positive and negative situations are presented as approximately equal in numeric magnitude. Secondly, because the outcomes differ in sign (positive, negative), the domain is no longer constant as it is in framing tasks (Fagley, 1993).With these considerations in mind, we wanted to investigate the influence of self-esteem on a reflection-type task.

\section{Method}

\section{Participants and design}

Sixty-three individuals participated in our experiment including 34 females and 29 males. Each participant received credit in their introductory psychology class for their participation. The experimental design included a between subjects factor of self-esteem 2 (high, low) and a within subjects factor of domain (gain, loss).

\section{Materials and procedure}

We first assessed individual levels of self-esteem by asking participants for their ratings on the Rosenberg self-esteem scale (Rosenberg, 1965). After completion of the self-esteem scale, participants were then presented with the gain and loss decision problems. To test our hypothesis for reflection tasks, we used a vignette developed by Tversky and Kahneman (1981). In this task participants are provided with the following: imagine that you face the following pair of concurrent decisions. First, examine both decisions, and then indicate the options that you prefer. They were then given the following decision choices:

\section{Decision 1:}

A. A sure gain of $\$ 240$.

B. $25 \%$ chance to gain $\$ 1000$, and $75 \%$ chance to gain nothing. Decision 2:

A. A sure loss of $\$ 750$

B. $75 \%$ chance to lose $\$ 1000$, and $25 \%$ chance to lose nothing.

After participants had made their choices, they were asked to turn the paper face down and were then debriefed about the experiment. 
Table 5. Study 3: Number of risk-avoidant and risk-seeking choices as a function of self-esteem, and domain

\begin{tabular}{lrr}
\hline & & Choices \\
\cline { 2 - 3 } & \multicolumn{1}{c}{ Risk-a voidant } & $\frac{\text { Risk-seeking }}{n}$ \\
\cline { 3 - 3 } & $N(\%$ total $)$ & $N(\%$ total $)$ \\
\hline High self-esteem & & $5(15)$ \\
Domain: & $29(85)$ & $23(68)$ \\
Gains & $11(32)$ & \\
Losses & & $8(28)$ \\
Low self-esteem & $21(72)$ & $27(93)$ \\
Domain: & $2(7)$ & \\
Gains & & \\
Losses & & \\
\hline
\end{tabular}

\section{Results and discussion}

To examine whether both gain and loss domain conditions produced the predicted reflection effect we first performed a chi-square analysis with participants' decisions acting as our dependent variable. This analysis yielded the common reflection effect $F(1,61)=36.12$, $p<0.001$. Furthermore, this effect was present in both the gains $X^{2}(1, N=63)=23.19, p<0.001$ and losses conditions $X^{2}(1, N=63)=23.19, p<0.001$.

Next, we examined whether level of self-esteem had an influence on participants' decisions.8,9 Therefore, using self-esteem as our independent variable, we performed a nominal logistic regression on participants' responses within each of the gain/loss conditions. The results of this analysis demonstrated that, similar to Study 2, LSE and HSE individuals differed significantly in their choices within the losses condition $X^{2}(1, N=63)=5.18, p<0.05$. As may be seen in Table 5, LSE individuals were more likely to choose the risky option and HSE individuals the risk-averse option. Next, we performed the same type of analysis for the gains condition. The results revealed that there was no significant effect for level of self-esteem $\mathrm{X}^{2}(1, \mathrm{~N}=63)=1.54, \mathrm{p}>0.2$.

Similar to the results of Study 2 regarding negative framing, our findings in Study 3 suggest that, when making choices in the loss domain, HSE and LSE individuals differ in their choice preference. As was the case in Study 2, both HSE and LSE individuals demonstrated a risk-seeking trend. However, compared to HSE individuals, LSE individuals were more likely to choose the risky option. Both studies provide converging evidence that LSE individuals are especially sensitive to negative frames. Consequently, they exhibit a greater magnitude of risky choices in the negative domain relative to their HSE counterparts.

\section{GENERAL DISCUSSION}

An issue that has plagued scholars for many ages is how to gain a better understanding of human rationality. An essential element to the ideal of human rationality is that of being "human." One central aspect of being human is that we possess an intrinsic part of our being that we refer to as "self" How we view our self, in a positive or negative light, can have profound effects upon many different aspects of our lives including the information that is available to us. And this "availability" of information can have profound effects upon situations, interactions and decisions that we encounter in the world around us.

The suggestion that information may be more or less "accessible” and in turn, influential, has historical precedence in psychology, most notably with work by Bruner (1957). The notion that individuals may vary in accessibility of certain types of information has ties to many areas of personality research. LSE and HSE individuals vary in their accessibility of information, with LSE having relatively more negative information accessible. And it is this difference in accessibility that leads to differences in frame imposition 
and sensitivity. And it is this difference in accessibility that leads to differences in frame imposition and sensitivity.

The propensity to reflect one's own internalized self views onto external information is an important issue for decision-making researchers, especially those investigating framing (e.g., Kuhberger, 1998; Lauriola et al., 2005; Levin et al., 2002; Reyna, Lloyd, \& Brainerd, 2003). Consideration of these findings should lead to a more precise understanding of underlying processes and better prediction of individual decisions. In our study, low self-esteem participants had a relatively greater tendency to perceive an ambiguous task more negatively, were more sensitive to negative frames and consequently, made more risky decision when the problem was framed in a negative way.

\section{Implications for theories on framing}

As Tversky \& Kahneman (1981) point out, there will be individual differences that affect the coding of alternatives as gains or losses. However, once these individual differences in encoding are identified, individuals should adhere to prospect theory predictions. Our findings demonstrate that, LSE individuals are more likely to perceive a negative frame and consequently, are more likely to pursue the risk-seeking option. Thus, this supports prospect theory predictions that individuals tend to be more risk seeking for alternatives that are coded as losses. Therefore, we believe that we have identified one personality component that may speak to some of the weak findings across framing studies (Kuhberger, 1998).

\section{Dual-process approaches to framing}

One way of understanding the framing process is by taking a dual-process approach (e.g., McElroy \& Seta, 2003, Stanovich \& West, 2000; Wang, in press). Deriving their central elements from classic dual-process models (e.g., Chaiken, 1987; Epstein, Lipson, Holstein, \& Huh, 1992; Fiske \& Neuberg, 1990; Petty \& Cacioppo, 1986; Sloman, 1996) the central theme in these dual-process models is that one route of processing performs decision-making in a more analytic, logical fashion and is less sensitive to contextual cues. The other route of processing is more automatic and reliant upon context, making it more likely to produce framing effects when utilized. The findings from our study suggest that personality factors, in our case self-esteem, may influence processing within the system responsible for framing (e.g., Stanovich \& West system 1). It would seem that, from the dual-process approach, LSE individuals are more likely to automatically contextualize negativity from the decision problem (Stanovich \& West, 2000), perceive negative emotion (Wang, in press), or be more likely to perceive negativity in contextual referencing (McElroy \& Seta, 2003).

Another theory that has been proposed as an explanation for why framing effects occur is fuzzy trace theory (e.g., Reyna \& Brainerd, 1991; Reyna \& Brainerd, 1995; Reyna, Lloyd, \& Brainerd, 2003). Fuzzy trace is governed by principles of memory retrieval. Retrieval for a given task can occur along a continuum that ranges from verbatim to gist like retrieval. Verbatim retrieval is more precise and involves a more objective approach to the problem whereas gist retrieval relies upon long-term memory. According to fuzzy trace theory, people will rely on as much "gist like" principles and this reliance on gist processing accounts for framing effects. Research investigating Fuzzy trace theory has shown that only the most general information is needed for individuals to make decisions, suggesting that the values in long-term memory are applied to representations of the problem to produce framing (Reyna \& Brainerd, 1991).

One implication from our findings concerns fuzzy-trace theory’s description of gist content. According to fuzzy-trace theory, "values, preferences and emotion-laden attitudes,"' make up the fuzzy-gist's that are represented in long-term memory (Reyna, Lloyd, \& Brainerd, 2003). And because long-term memory is derived from experience, it seems reasonable that the experiences of LSE and HSE individuals may differ. More specifically, HSE individuals may be experiencing more positive or unbiased information within their interactions with the world. The long-term memory of LSE individuals however, may be negatively biased thus, increasing the likelihood that the gist retrieval will be relatively more negative.

\section{Potential limitations}

In our studies, although participants were asked to make a decision, the implementation of their decision would be carried out by someone else. Thus, they knew that they would have no control over the success or failure of the option that they chose. Because of this, differences in participants' level of self-esteem should 
have had a minimal influence on the likelihood of each options potential success or failure. If our situation did implicate the abilities of our participants and if our participants thought that they would personally carry out their decision, then different results might have been obtained. In situations in which individuals do actually implement their decisions, other factors come into play. For example, HSE individuals may persevere in making self-defeating decisions because of their elevated sense of egotism (Zhang \& Baumeister, 2006). However, it is important to note that, although the options we used in the present studies did not involve the ability of our decision-makers, their level of self-esteem nevertheless acted as a perceptual lens that colored their decisions via the way they framed the problem.

\section{Prior research and future directions}

When compared to the self-framing studies of Wang (2004), our findings yield an interesting contrast. Overall, both sets of findings are similar, in that, both found results consistent with typical framing effects. However, the Wang study found only a moderate framing effect whereas we found significant and seemingly stronger framing in our study. This is not surprising in light of the finding by Kuhberger (1998) that the Asian disease problem yields the strongest framing effect among the many variations of the risky-choice decision problems. In light of this, we believe that this study provides more validity for the self-framing methodology and will hopefully encourage more research with this technique.

Some of our findings also were different from the Wang studies. Across both studies, Wang found a generally positive hedonic tone, with this positivity being greater in the life-death than monetary scenario. This is particularly interesting in light of our finding that about two-thirds of our participants demonstrated a negative hedonic tone when they self-framed the task. In fact, the mean for the Asian disease self-framing task we used was 2.28, substantially below the neutral score of 3 . One potential explanation may lie in the nature of the task. It may be that the Asian disease problem lends itself to relatively more negative self framing. However, this remains speculative and an interesting question for future research.

Our research focused on how self-esteem influenced traditional risky-choice framing and reflection decision tasks. Further research is needed to determine whether our findings will generalize across different types of decision-making situations. For example, tasks that are more related to the self may enhance the potential influence of self-esteem in decision-making whereas those that are more distant may attenuate this influence. An examination of issues such as this should help provide a fuller understanding of framing effects. 


\section{APPENDIX 1}

Imagine that the U.S. is preparing for the outbreak of an unusual Asian disease, which is expected to kill 600 people. Two alternative programs to combat the disease have been proposed. Assume that the exact scientific estimates of the consequences of the programs are as follows:

If Plan $\mathbf{A}$ is adopted,
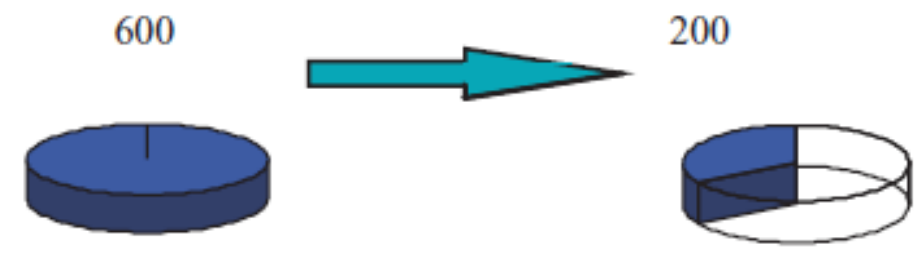

If Plan B is adopted, there is a one-third chance,

600

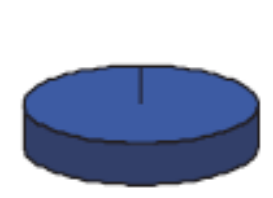

600
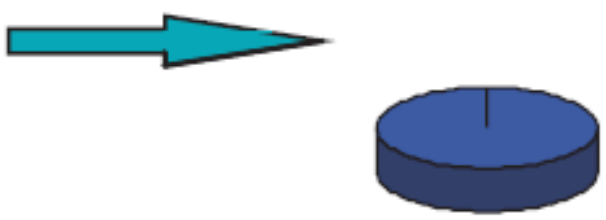

and a two-thirds chance
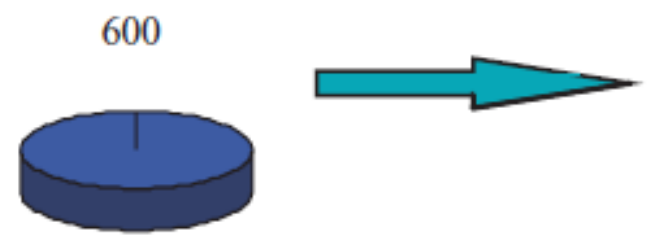

0

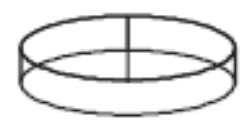

Based on your interpretation of the pie display of the expected outcomes, complete the following sentences in your own words:

If Plan A is adopted, people will

If Plan B is adopted, there is a one-third chance that people will and a twothirds chance that people will

Given the previous information, which program would you choose?

Program A

Program B 


\section{APPENDIX 2}

Mean hedonic ratings and total frequency counts for self-imposed frames

\begin{tabular}{lcr}
\hline Frame descriptor & Mean rating & Frequency \\
\hline Be saved & 4.79 & 27 \\
Cured & 4.79 & 14 \\
Live & 4.57 & 82 \\
Survive & 4.36 & 37 \\
Guaranteed & 4.07 & 3 \\
Lost & 1.5 & 17 \\
Be killed & 1.29 & 6 \\
Die & 1.14 & 252 \\
\hline
\end{tabular}

\section{NOTES}

1We found that in our sample 32 participants self-imposed a mixed frame.

2While participants varied in the format of the numerical representations of the percentage probabilities derived from the diagram (e.g., $200,1 / 3,0.33$ ), all but one participant interpreted it in such a way that the two alternatives had equal expected values and this person imposed a mixed frame. Three other participants focused on the "empty”' section of our diagram (e.g. 2/3 saved). Excluding these participants did not affect the significance of our analysis.

3There were approximately equal numbers of LSE (14) and HSE (18) participants who imposed a mixed frame.

4For this and all subsequent nominal logistic analyses having two or more independent variables, we performed the analysis using simultaneous entry.

5For tables and analyses using the high/low self-esteem division we performed a median split. As was the case in Study 1 , 32 or below represented low and 33 or higher represented high.

6Four participants either did not follow instructions correctly or failed to complete this section. Therefore they were not included in our analyses examining affect.

7Special thanks to anonymous reviewer 3 for clarification on this topic.

8As was the case in the two previous studies we obtained a median split of 32 and below for LSE and 33 and above for HSE participants. 9As was the case in studies 1 and 2 gender was divided relatively well with the HSE condition having 16 males and 18 females and the LSE condition having 16 females and 13 males. Because there is no research pointing to potential gender differences in the reflection effect we did not analyze gender in this study.

\section{ACKNOWLEDGEMENTS}

The authors thank Cathy Seta and Hakan Ersever for their helpful input on this paper.

\section{REFERENCES}

Bartels, D. M. (2006). Proportion dominance: The generality and variability of favoring relative savings over absolute savings. Organizational Behavior and Human Decision Processes, 100, 76-95.

Baumeister, R. F. (1993). Self-esteem: The puzzle of low self-regard. New York, NY: Plenum Press.

Bellavia, G., \& Murray, S. (2003). Did I do that? Self esteem-related differences in reactions to romantic partner's mood. Personal Relationships, 10, 77-95.

Bless, H., Betsch, T., \& Franzen, A. (1998). Framing the framing effect: The impact of context cues on solutions to the Asian disease problem. European Journal of Social Psychology, 28, 287-291.

Bloomfield, A. (2006). Group size and the framing effect: Threats to human beings and animals. Memory and Cognition, 34, 929-937. 
Bruner, J. S. (1957). On perceptual readiness. Psychological Review, 64, 123-152.

Chaiken, S. (1987). The heuristic model of persuasion. In M. P. Zanna , J. M. Olson , \& C. P. Herman (Eds.), Social influence: The Ontario Symposium (Vol. 5, pp. 3-39). Hillsdale, NJ: Erlbaum.

Chatterjee, S., Heath, T. B., Milberg, S. J., \&France, K. R. (2000). The differential processing of price in gains and losses: The effects of frame and need for cognition. Journal of Behavioral Decision Making, 13, 61-75.

Clark, H., \& Chase, W. (1972). On the process of comparing sentences against pictures. Cognitive Psychology, 3, 472517.

Coopersmith, S. (1967). The antecedents of self-esteem. San Francisco: Freeman.

Elliott, C. S., \& Archibald, R. B. (1989). Subjective framing and attitudes towards risk. Journal of Economic Psychology, 10, 321-328.

Epstein, S., Lipson, A., Holstein, C., \& Huh, E. (1992). Irrational reactions to negative outcomes: Evidence for two conceptual systems. Journal of Personality and Social Psychology, 38, 889-906.

Fagley, N. S. (1993). A note concerning reflection effects versus framing effects. Psychological Bulletin, 113(2), 451452.

Fagley, N. S., \& Miller, P. M. (1987). The effects of decision framing on choice of risky vs. certain options. Organizational Behavior and Human Decision Processes, 39, 264-277.

Fagley, N. S., \& Miller, P. M. (1990). The effect of framing on choice: Interactions with risk-taking propensity, cognitive style, and sex. Personality and Social Psychology Bulletin, 16, 496-510.

Fagley, N. S., \& Miller, P. M. (1997). Framing effects and arenas of choice: Your money or your life? Organizational Behavior and Human Decision Processes, 71, 355-373.

Fiske, S. T., \& Neuberg, S. L. (1990). A continuum model of impression formation: From category-based to individuating processes as a function of information, motivation, and attention. In M. P. Zanna (Ed.), Advances in experimental social psychology (Vol. 23 ). Hillsdale, NJ: Erlbaum.

Gilbert, D. T. (1991). How mental systems believe. American Psychologist, 46, 107-119.

Hoyle, R. H., Kernis, M. H., \& Leary,M. R. (1999). Selfhood: Identity, esteem, regulation. Boulder, CO:Westview Press.

Igou, E. R., \& Bless, H. (in press). On Undesirable Consequences of Thinking: Framing Effects as a Function of Substantive Processing. Journal of Behavioral Decision Making. Revision in progress.

Kahneman, D., \& Tversky, A. (1979). Prospect theory: An analysis of decision under risk. Econometrica, 47, $263-291$.

Kling, K. C., Hyde, J. S., Showers, C. J., \& Buswell, B. N. (1999). Gender differences in self-esteem: A meta-analysis. Psychological Bulletin, 125, 470-500.

Koch, E. J. (2002). Relational schemas, self-esteem, and the processing of social stimuli. Self and Identity, 1, $271-279$. Ku"hberger, A. (1998). The influence of framing on risky decision: A meta-analysis. Organizational Behavior and Human Decision Processes, 75, 23-55.

Lauriola, M., \& Levin, I. P. (2001). Personality traits and risky decision-making in a controlled experimental task: An exploratory study. Personality and Individual Differences, 31, 215-226.

Lauriola, M., Russo, P. M., Lucidi, F., Violani, C., \& Irwin, P. (2005). The role of personality in positively and negatively framed risky health decisions. Personality and Individual Differences, 38, 45-59.

Leary, M. R., \& Baumeister, R. F. (2000). The nature and function of self-esteem: Sociometer theory. In M. P. Zanna (Ed.), Advances in experimental social psychology (pp. 1-62). San Diego, CA: Academic Press.

LeBoeuf, R. A., \& Shafir, E. (2003). Deep thoughts and shallow frames: On the susceptibility to framing effects. Journal of Behavioral Decision Making, 16, 77-92. 
Levin, I. P., Gaeth, G. J., Schneider, S. L., \& Lauriola, M. (2002). A new look at framing effects: Distribution of effect sizes, individual differences, and independence of types of framing. Organizational Behavior and Human Decision Processes, 88, 411-429.

Levin, I. P., Schneider, S. L., \& Gaeth, G. J. (1998). All frames are not created equal: A typology and critical analysis of framing effects. Organizational Behavior \& Human Decision Processes, 76(2), 149-188.

McElroy, T., \& Seta, J. J. (2003). Framing effects: An analytic-holistic perspective. Journal of Experimental Social Psychology, 39, 610-617.

McElroy, T., \& Seta, J. J. (2004). On the other hand am I rational? Hemispheric activation and the framing effect. Brain and Cognition, 55, 572-580.

McElroy, T., \& Seta, J. J. (2006). Does it matter if it involves my group? How the importance of collective-esteem influences a group-based framing task. Social Cognition, 24, 319-333.

Miller, P. M., \& Fagley, N. S. (1991). The effects of framing, problem variations, and providing rationale on choice. Personality and Social Psychology Bulletin, 17, 517-522.

Petty, R. E., \& Cacioppo, J. T. (1986). Communication and persuasion: Central and peripheral routes to attitude change. New York: Springer-Verlag.

Reyna, V. F., \& Brainerd, C. J. (1991). Fuzzy-trace theory and framing effects in choice: Gist extraction, truncation, and conversion. Journal of Behavioral Decision Making, 4, 249-262.

Reyna, V. F., \& Brainerd, C. J. (1995). Fuzzy-trace theory: An interim synthesis. Learning and Individual Differences, 7, $1-75$.

Reyna, V. F., Lloyd, F. J., \& Brainerd, C. J. (2003). Memory, development, and rationality: An integrative theory of judgment and decision making. In S. L. Schneider , \& J. Shanteau (Eds.), Emerging perspectives on judgment and decision research (pp. 201-245). New York, NY, US: Cambridge University Press.

Rosenberg, M. (1965). Society and the adolescent self-image. Princeton, NJ: Princeton University Press. Rosenberg, M. (1979). Conceiving the self. New York: Basic Books.

Schlenker, B. R. (1980). Impression management. Monterey, CA: Brooks/Cole.

Schneider, S. L. (1992). Framing and conflict: Aspiration level contingency, the status quo, and current theories of risky choice. Journal of Experimental Psychology: Learning, Memory, and Cognition, 18, 1040-1057.

Sieck,W., \& Yates, J. F. (1997). Exposition effects on decision making: Choice and confidence in choice. Organizational Behavior and Human Decision Processes, 70, 207-219.

Simon, A. F., Fagley, N. S., \& Halleran, J. G. (2004). Decision framing: Moderating effects of individual differences and cognitive processing. Journal of Behavioral Decision Making, 17, 77-93.

Sloman, S. A. (1996). The empirical case for two systems of reasoning. Psychological Bulletin, 119, 3-22.

Smith, S. M., \& Levin, I. P. (1996). Need for cognition and choice in framing effects. Journal of Behavioral Decision Making, 9, 283-290.

Stanovich, K. E., \& West, R. F. (1998). Individual differences in rational thought. Journal of Experimental Psychology: General, 127, 161-188.

Stanovich, K. E., \& West, R. F. (2000). Individual differences in reasoning: Implications for the rationality debate. Behavioral and Brain Sciences, 23, 645-726.

Sullivan, H. S. (1953). The interpersonal theory of psychiatry. New York: W. W. Norton.

Takemura, K. (1994). Influence of elaboration of the framing of decision. The Journal of Psychology, 128, 33-39.

Trzesniewski, K. H., Donnellan, M. B., \& Robins, R. W. (2003). Stability of self-esteem across the life span. Journal of Personality and Social Psychology, 84, 205-220. 
Tversky, A., \& Kahneman, D. (1981). The framing of decisions and the rationality of choice. Science, 221, 453-458.

Wang, X. T. (2004). Self-framing of risky choice. Journal of Behavioral Decision Making, 17, 1-16.

Wang, X. T. (in press). Emotions within reason: Resolving conflicts in risk preference. Cognition and Emotion.

Wang, X. T., Simons, F., \& Bre'dart, S. (2001). Social cues and verbal framing in risky choice. Journal of Behavioral Decision Making, 14, 1-15.

Watson, D., Clark, L. A., \& Tellegen, A. (1988). Development and validation of brief measures of positive and negative affect: The PANAS scales. Journal of Personality and Social Psychology, 54, 1063-1070.

Zhang, L., \& Baumeister, R. F. (2006). Your money or your self-esteem: Threatened egotism promotes costly entrapment in losing endeavors. Personality and Social Psychology Bulletin, 32, 881-893.

Zhang, Y., \& Buda, R. (1999). Moderating effects of need for cognition on responses to positively versus negatively framed advertising messages. Journal of Advertising, 28, 1-15. 